\title{
Evaluation of Routing Protocols and Mobility in Flying Ad-hoc Network
}

\author{
Emad Felemban \\ College of Computer \& Information Systems, \\ Umm Al-Qura University, Makkah, Saudi Arabia
}

\begin{abstract}
The ability of dynamic reconfigurability, quick response and ease of deployment has made Unmanned Aerial Vehicles (UAVs), a paramount solution in several areas such as military applications. Flying ad-hoc network (FANET) is a network of UAVs connected wirelessly and configured continuously without infrastructures. Routing on its own is not significant, but the mobility sequence of a UAV in FANETs is a more significant factor and an interesting research topic. The routing protocols gives us a certain and better perception of routing structure for FANETs. In this paper, routing protocols such as Ad-hoc On-Demand Vector (AODV), Dynamic Source Routing (DSR), Temporally Ordered Routing Algorithm (TORA), Geographic Routing Protocol (GRP) and Optimized Link State Routing (OLSR) are compared using performance parameters such as number-of-hops, packet loss ratio, throughput, end-to-end delay and throughput. The mobility models like Pursue Mobility Model (PRS), Semi-Circular Random Movement (SCRM), Manhattan Grid Mobility Model (MGM) and Random Waypoint Mobility (RWPM). The evaluation is carried out with three scenarios including one sender node and one receiver node, all senders one receiver and all senders all receivers are considered for above protocols and mobility models. For all evaluation scenarios, the performance of OLSR is the most efficient among the five routing protocols under four different performance parameters due to its proactive nature which makes the routing information up to date with the help of MPR (Multi Point Relay) in the network, resulting in the reduction of routing overhead in the network.
\end{abstract}

Keywords-Flying ad-hoc network (FANET); mobility models; adhoc routing protocols; OPNET; Unmanned Aerial Vehicles (UAVs)

\section{INTRODUCTION}

Current development in UAVs is offering different opportunities at reasonable price. As a result, the ability of dynamic reconfigurability, quick response and ease of deployment has enabled UAVs to be a paramount solution in several applications [1][2]. Although UAVs are advantageous, high mobility is essential for the networks which need adequate control over UAVs. FANETs belong to an important class of UAVs, where many UAVs are connected in ad-hoc manner. UAV networking was given a new terminology due to modernization of Internet of Things (IoT), but it has the same functioning as Internet of Drones (IoD) shown in Fig. 1. IoD is a layered network control architecture for coordinating unmanned aerial vehicles to control airspace for navigation services.

As opposed to the traditional (MANET), the main feature of FANET is the highly dynamic scenario, which signifies that nodes move in higher mobility and in the meantime the network topology changes more quickly, so protocols under such circumstances are more challenging. The main advantage of the FANET networks is not to depend on a physical infrastructure; each node will be able to access the nodes through its closer resources, trusting the cooperation and collaboration of the other nodes and this get through them to their destination. Typical examples are applications that range from networks in the armed forces, to the applications of business, to reach the applications in case of natural disaster or terrorist acts. FANET network will offer services like absence of centralization, ability to configure networks automatically, 3D mobility, ability to exploit the resources of a working group and comfort. The important research concerns are whether MANET routing protocols are suitable for FANET, which performs better in high dynamic scenarios and the effect of nodes mobility on network performance.

The stability of Ad-Hoc networks [4] is compromised due to inadequate protocols. We can achieve improved efficiency in the passage of information in time and quality but achieving transparency is challenging. The end user does not have to make a different connection type according to the type of network (fixed or mobile) to extend them to a greater number of users [5]. In addition, due to wireless and dynamic nature of Ad-hoc networks, there is packet loss due to transmission error.

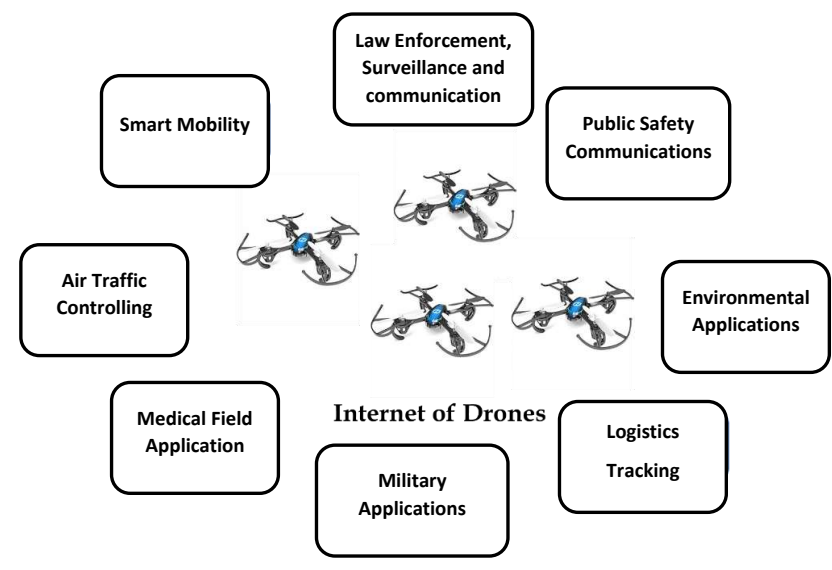

Fig. 1. A Summary of Internet of Drones (IoD) Application Services [3].

FANETs are a group of UAVs that can perform the different functions without human service. It is a network without infrastructure that has an autonomous set of mobile nodes that collaborate together to share information [6]. These mobile 
nodes can be constituted in any network topology as router through wireless communication. It has the ability to connect to the Internet or the cellular wireless network [7]. Each device in a FANET has the freedom to fly freely in all directions and it can alter its connections to other nodes constantly. Each node must forward traffic not related to itself. Consequently, and because of this it can function as a router [8]. FANET topology continues to change due to the mobility of the UAVs, it make them less secure networks. To overcome the threats it is crucial to use some security technologies in the network. For an adhoc network, not every node could be within the transmission reach between them, therefore nodes are compelled to forward network traffic on behalf of other nodes. For instance, we can contemplate a simple scenario, two nodes, a sender node (S) sends data to the destination node (D), which is three jumps away, and the data traffic will arrive at its destination. The procedure of forwarding network traffic from sender to destination is called routing. FANETs can assist networks which are operated to enable wireless connectivity in the places where deployment of physical infrastructure is hard or costly.

During the research in modeling FANET networks, it was possible to verify the importance of protocol evaluation. There is a need to model FANETs and analyze the performance of proactive and reactive routing protocols. Due to several applications, FANETs have captivated several research institutions and automotive industries. The main problem is the implementation of a proper routing mechanism as the result of various issues. Routing on its own is not significant, but the mobility sequence of a UAV in FANETs is also a more significant factor. Ad-Hoc routing protocols are categorized into many different classifications, one of which is topology based. It is analyzed that these routing protocols gives us a better perception for routing structure of FANETs. The evaluation of routing protocols is required using different mobility models for FANETs.

The main objective of this paper is to introduce FANETs and compare existing routing protocols (OLSR, AODV, DSR, TORA and GRP) and mobility models (RWPM, MGM, SCRM and PRS) for FANETs. The performance parameters include end to end delay, packets loss ratio, number of hops and throughput. The affect of speed over network performance is also evaluated. The rest of the paper is organized as follows. A detailed literature review is provided for routing protocols and mobility models in Section II. The Section III comprises of performance evaluation. Section IV draws conclusion with future work.

\section{Literature ReVIEW}

Wireless networks are formed by connecting radio transmitters to electronic devices (computers, smartphones, balloons, drones, etc.), with the support of a central access point to manage the network. Wireless standards include detailed requirements for the data link layer, physical layer and medium access control (MAC) regarding terminology introduced by IEEE 802.11 standards. There are many essential characteristics of wireless networks including high mobility, flexible communication system, simplicity and scalability.

Generally, The wireless network is divided in two classes based on their infrastructure as can be seen in Fig. 2. A network that has fixed base stations is called infrastructurebased network. These networks accommodate coordination among the mobile UAVs [9]. Infrastructure-less networks does not have any well defined infrastructure. They are a group of dynamically situated UAVs that keep the interconnections among UAVs by changing topology.

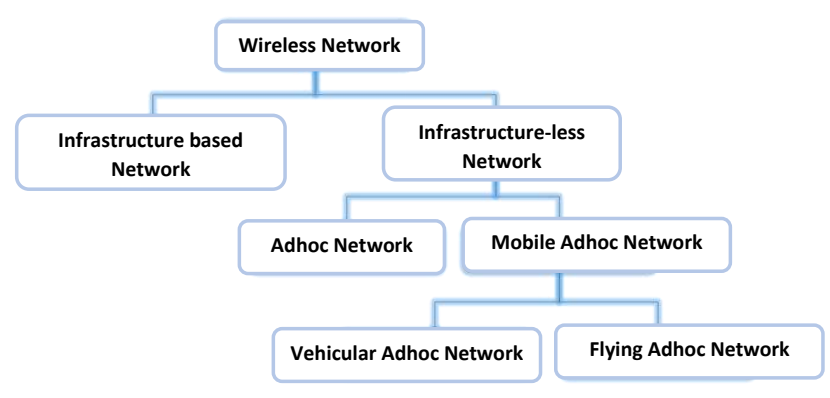

Fig. 2. Wireless Network Classifications.

The background of Ad-Hoc networks is related to the creation of the PRNET (Packet Radio Network) by the DoD (US Department of Defense) in 1972. The aim was to connect a network of computers to provide packet exchanges to mobile elements used in the battlefield e.g soldiers, aircraft, etc. The current proliferation of portable devices and the diffusion of wireless technology have permitted ad-hoc wireless networks to thrive. Moreover, interest in communication among ad-hoc wireless networks has raised consistently [9]. Ad-hoc network can be formed when you want to exchange data in the absence of a centralized access point (access point): the network is formed "spontaneously". The advantage of being free from any infrastructure constraints helps these networks in obtaining an intrinsic ease and speed for installation [10]. Each node of adhoc network can act as a router. A node, instead of directly sending the packets to the recipient node, can route the packets to another node within its radio visibility.

In air-to-air wireless communications, UAVs are connected to each other in ad-hoc manner. These UAVs are independent of any restriction on transmission ranges between them [14]. In air-to-ground-wireless communications, UAVs can extend their connectivity by communicating with infrastructures and improve quality of service [15]. In this paper, we have focused on air-to-air communication i.e infrastructure-less protocols. MANETs are a self-configuring infrastructure-less network [9]. Each device can relocate without restriction within network. Vehicular Ad-Hoc network is a derived class from MANETs (As shown in Table I). In VANET, vehicles are connected via wireless communication. VANET is a distributed and selfformation network [11]. VANET can create new systems to provide safety and comfort in toll-tax payment to save time. FANETs can be described as a group of Unmanned Aerial Vehicles, which communicate with each other without the need of any access point, while at least one among the group acts as the server or satellite. These are automated systems, which don't involve any human input for their basic operations [12]. All activity of the network including the discovery of its topology or the sending of messages is carried out by the nodes that integrate it. This type of network has arbitrary topology. The main consequence of this mobility is that links can be formed and broken frequently, which implies that the network 
TABLE I. The MAIn Differences Between MANETs, VANETs, AND FANETS [13]

\begin{tabular}{|c|c|c|c|}
\hline $\begin{array}{c}\text { Types } \\
\text { Parameters }\end{array}$ & MANET & VANET & FANET \\
\hline $\begin{array}{c}\text { Node } \\
\text { Mobility }\end{array}$ & $\begin{array}{c}\text { Low } \\
\text { Compactness }\end{array}$ & $\begin{array}{c}\text { Medium } \\
\text { Compactness }\end{array}$ & $\begin{array}{c}\text { High } \\
\text { Compactness }\end{array}$ \\
\hline $\begin{array}{l}\text { Mobility } \\
\text { Model }\end{array}$ & Random & Regular & $\begin{array}{c}\text { Regular } \\
\text { for } \\
\text { pre } \\
\text { determined } \\
\text { paths }\end{array}$ \\
\hline $\begin{array}{c}\text { Node } \\
\text { Density }\end{array}$ & Low & Medium & Very Low \\
\hline $\begin{array}{l}\text { Topology } \\
\text { Change }\end{array}$ & Slow & $\begin{array}{l}\text { Average } \\
\text { Speed }\end{array}$ & Fast \\
\hline $\begin{array}{c}\text { Radio } \\
\text { Propagation } \\
\text { Model }\end{array}$ & $\begin{array}{l}\text { Near to } \\
\text { earth (2D) }\end{array}$ & $\begin{array}{l}\text { Near to } \\
\text { earth (2D) }\end{array}$ & $\begin{array}{l}\text { Over to } \\
\text { earth (3D) }\end{array}$ \\
\hline $\begin{array}{c}\text { Power } \\
\text { Consumption }\end{array}$ & $\begin{array}{l}\text { Energy } \\
\text { efficient } \\
\text { protocols }\end{array}$ & $\begin{array}{l}\text { Not } \\
\text { required }\end{array}$ & $\begin{array}{c}\text { Energy } \\
\text { efficacy } \\
\text { for mini } \\
\text { UAV \& } \\
\text { small UAVs }\end{array}$ \\
\hline $\begin{array}{c}\text { Computat } \\
\text { ional } \\
\text { Power } \\
\end{array}$ & Limited & $\begin{array}{c}\text { Above } \\
\text { average }\end{array}$ & $\begin{array}{c}\text { Above } \\
\text { average }\end{array}$ \\
\hline
\end{tabular}

must be self-organized. The path between an origin and a certain destination that crosses several intermediate nodes can be modified and When this happens, the network must be able to re-organize itself to establish new path in least possible time. This functionality requires that each member of the network will be able to forward data on behalf of other members act as a router. The nodes of a FANET network have components or associated factors that must be considered when carrying out an efficient routing. These factors include auto configuration, bandwidth, distance, energy, routing path, partitions/unions, speed and variation in the routing path. The FANET networks are based on the concept of Peer-to-Peer (P2P) where peers are mostly UAVs.

The routing protocols can be classified into five major categories including static routing, position-based routing and Hierarchical routing, position-based routing, swarm-based routing, hierarchical routing and topology-based routing. Static routing protocols follow a static routing table loaded in the UAV's during the mission which cannot be changed further. The protocols cannot update the table dynamically in case of failure so they are not suitable for dynamic networks. Static protocols are further categorized in swarm intelligence and topology based routing protocols. Swarm based routing protocols are inspired from behavior of natural organisms. The algorithm in swarm intelligence is motivated by the behavior of different insects such as ants, bees, fireflies and fish etc. Swarm intelligence based algorithms offer efficient solutions for UAVs. The authors in [17] [18] presents Bee Ad-Hoc and APAR. Position based routing protocols establish route between UAVs using their geographical locations. Each UAV can use either hierarchical position, grid position or reactive position service. The examples include Delay Tolerant (DTN), Non-DTN and Heterogeneous Protocols [19]. The hierarchical protocols are other set for routing solution for FANET, which are founded in accordance with addressing the network scalability problem where the network contains of numerous clusters of different mission zones. The hierarchical protocols for FANET include clustering algorithm and mobility prediction clustering. In the clustering algorithm the network includes over many clusters. Every cluster has a cluster head $(\mathrm{CH})$. All nodes in the cluster can directly communicate with cluster head. This type of model may generate improved performance of outcomes when the number of UAVs is higher and the mission range is large [20]. The mobility prediction algorithm can solve this issue by making cluster updates frequently. It uses a tree structured prediction algorithm [21].

The topology-based routing protocols are classified into reactive, proactive and hybrid routing. Reactive Routing is suitable for FANETS in the discovery of a path between nodes which is unavailable for UAVs. This kind of routing also leads to high latency. Multicast Ad hoc On-Demand Distance Vector (M-AODV) is an example of reactive routing [16]. Proactive routing tables are constantly shared periodically among nodes to assure availability of routing paths among UAVs. For example OLSR, Directional Optimized Link State Routing Protocol (D-OLSR), Cartography Enhanced OLSR (CE-OLSR) and DSDV [16] belong to this category. Hybrid routing solves the high latency problem of reactive protocols. The examples of this type are Hybrid Wireless Mesh Protocol (HWMP), GRP and TORA.

This paper is committed to the most critical topologybased routing protocols recommended for FANETs namely AODV, DSR, TORA, GRP and OLSR. AODV was introduced as an evolution of DSDV. It deals with sequence numbers and routing tables. It also provides on-demand routing as it saves information of nodes that intervene in data transmission. This protocol keeps all routes in cache memory when they are required and discard them when they are not needed [22] [23].

The DSR protocol follows routing at source. The nodes having a destination maintains cache to save list of nodes in this path. The methods such as detection and route maintenance are responsible to provide updates as new routes are learnt. It has on demand features but it is not table-driven. The UAV sends a packet to specify the route. The entire path information is put in the packet by the source [23]. It differs from table-driven and link-state routing. It collects the addresses of all the middle nodes between itself and the desired destination when finding routes.

The TORA protocol follows link reversal routing. It maintains a directed graph from source to destination without presence of any cycle. The network load is minimized in TORA and it keeps the shortest route. It is considered an efficient protocol because it does not saturate network traffic unnecessarily [25] [26]. If a node wants to know the path to destination, it will broadcast a query packet that propagates until it reaches a node having path to destination or the recipient node. The responding node is served by a User Datagram Protocol (UDP) that will add its weight and broadcasts it to allow all intermediate nodes to know about this modification.

GRP detects the location of nodes using global positioning system to obtain information about network. The route is discovered by source node and transfers data from source to destination using route. This concept is referred as hybrid routing protocol. There are several methods to find out the location of network nodes such as GPRS and GPS, etc. [24]. The transmission in this protocol is dependent on the neighbors located at one hop distance and destination node. The data is forwarded using greedy forwarding and face- 2 
TABLE II. FANET APPLICATIONS USING DIFFERENT MOBILITY MODELS [13].

\begin{tabular}{|c|c|c|}
\hline $\begin{array}{l}\text { Application } \\
\text { Class }\end{array}$ & $\begin{array}{c}\text { Mobility } \\
\text { Model }\end{array}$ & $\begin{array}{c}\text { Scenario } \\
\text { Description }\end{array}$ \\
\hline $\begin{array}{l}\text { Search and } \\
\text { rescue }\end{array}$ & $\begin{array}{l}\text { MGM } \\
\text { SRCM } \\
\text { RWPM }\end{array}$ & $\begin{array}{l}\text { Random exploration on } \\
\text { a definite target zone. } \\
\text { Scanning in a circular area } \\
\text { Each UAV chooses the scan } \\
\text { pattern in random location }\end{array}$ \\
\hline $\begin{array}{l}\text { Traffic and } \\
\text { urban } \\
\text { monitoring }\end{array}$ & $\begin{array}{l}\text { MGM } \\
\text { SRCM }\end{array}$ & $\begin{array}{c}\text { Surveillance of city roads } \\
\text { Patrolling of a crash event } \\
\text { before the rescue team reaches }\end{array}$ \\
\hline $\begin{array}{l}\text { Survey and } \\
\text { Patrolling }\end{array}$ & SRCM & Surveillance of a target \\
\hline $\begin{array}{l}\text { Target } \\
\text { tracking }\end{array}$ & $\begin{array}{l}\text { Pursue } \\
\text { (PRS) }\end{array}$ & $\begin{array}{c}\text { Crime tracking } \\
\text { Pursuing of a critical } \\
\text { moving target }\end{array}$ \\
\hline \multicolumn{3}{|c|}{$\begin{array}{l}\text { SRCM: Semi-Random Circular Movement, } \\
\text { PR: Pursue mode; MGM; Manhattan Grid Mobility, } \\
\text { RWPM: Random waypoint model }\end{array}$} \\
\hline
\end{tabular}

routing perimeter [21][23].

The OLSR protocol keeps the information about network in a table format in every node. Whenever a change take place in network, it triggers an update broadcast to inform all nodes about it. Consequently, it may cause packet overhead that affect throughput, energy and bandwidth of network. The main advantage is that each node is aware about routes to destination all the time without any route discovery mechanism but it faces performance issues in case of high mobility or large network size [26]. It utilizes hello, interface declaration (MID) and topology information control messages for its working.

The mobility models for FANETs are divided into four classes including MGM, RWPM, PRS and SCRM. MGM utilizes grid path topology (Fig. 3) and introduces a probable approach that whether a vehicle will keep on moving in same direction or change it [15]. In this model, the UAVs can move around in vertical and horizontal angles only. Although this model offers flexibility to change direction but puts geographic restrictions on node mobility. It is suitable in only a few UAVs scenarios.

In RWPM model, UAV begins movement by waiting in a place for a few times. Once it finishes, it selects a new random speed and location within a specified region [27]. This process is repeated with a short break everytime. Fig. 4 elaborates this model.

In PRS mobility model, the UAVs tracks a target which can be elaborated using this relation: Newlocation $=$ oldplace + randomvector + acceleration [target - oldplace]. The acceleration specifies the movement of UAVs in direction of specified target [28]. The random vector is an offset to measure movement of individual UAV. Fig. 5 shows detail of his model, we can see the randomness of all UAVs is controlled to maintain tracking.

SCRM is different from RWPM model due to its predetermined flight plan [29]. The route is hexagon in this model. The aircraft is placed in different locations within a square area and desired object is chosen as shown in Fig. 6. In this way, information is collected for simulating UAV's in curved movement scenarios (Table II).

\section{FANET SimUlation MODEL}

In this section, a detailed discussion is provided on available network simulators and our choice of simulator for experimentation. The design of FANET simulation model is elaborated. The evaluation and performance techniques are also presented. Three different scenarios are considered for performance evaluation.

\section{A. Network Simulators}

Before starting with the design of the network model, a search for network simulators was carried out to design and simulate network models in several viewpoints. The available softwares include MATLAB, OMNeT ++, NS-3, GNS3 and OPNET Modeler, which are well-known simulators in the area of telecommunications networks. NS-2 and NS-3 are open source programs. GNS-3 is open source but with graphic development. Few platforms are licensed but there are student versions that allow their use free of cost. For the OPNET simulation tool, it is commercial, and the source code is not open to everyone. However, OPNET has a broad integrated development environment to design and simulate network models. To carry out experimental evaluation, OPNET Modeler has been adopted based on the Microsoft Windows platform. The protocols available in the library are reactive, proactive and hybrid as compared to other commercial simulators. The OPNET Modeler simulation platform has several parameters in communication system environments especially wireless communications. For this work, FANET is evaluated for different routing protocols. OPNET provides graphical user interfaces known as editors to catch the specifications of installed networks, equipment, and protocols. The three essential editors are project, node and process editors. The network parameters to be measured are delay and performance. These are substantially affected by the algorithms of the routing protocols. Therefore, the parameters play a vital role in finding a suitable routing protocol for FANETs [30]. OPNET Modeler users can make customized models and simulate several scenarios. It is object-oriented and ensures a hierarchical approach to model communication networks. The performance parameters for FANETs include delay, throughput, packet hop count and packet loss using proactive and reactive routing protocols.

\section{B. Design of FANET Model}

The simulation scenarios are designed on the OPNET Modeler platform with help of different FANET network configuration parameters. The parameters such as profile, mobility and application configuration, wireless server and work stations are used in network design. Only 2D scenarios are discussed here. The selected protocols are AODV, DSR, TORA, OLSR and GRP. For the tests, OPNET simulator was used. Test scenarios were generated in area of $1500 \times 2000$ meters, in which 15 nodes were randomly arranged and the speed of nodes was set to 5 and $40 \mathrm{~m} / \mathrm{s}$. Furthermore, mobility models chosen were PRS, MGM, RWPM and SCRM. The nodes generated CBR traffic (Constant Bit Rate). The evaluated metrics are delay, transmission failures, packet hop count and throughput. There are other previous works where comparison tests were carried out between ad-hoc routing protocols. The traffic corresponding to the routing protocols will have a high priority so that routes can be formed more quickly. For this 


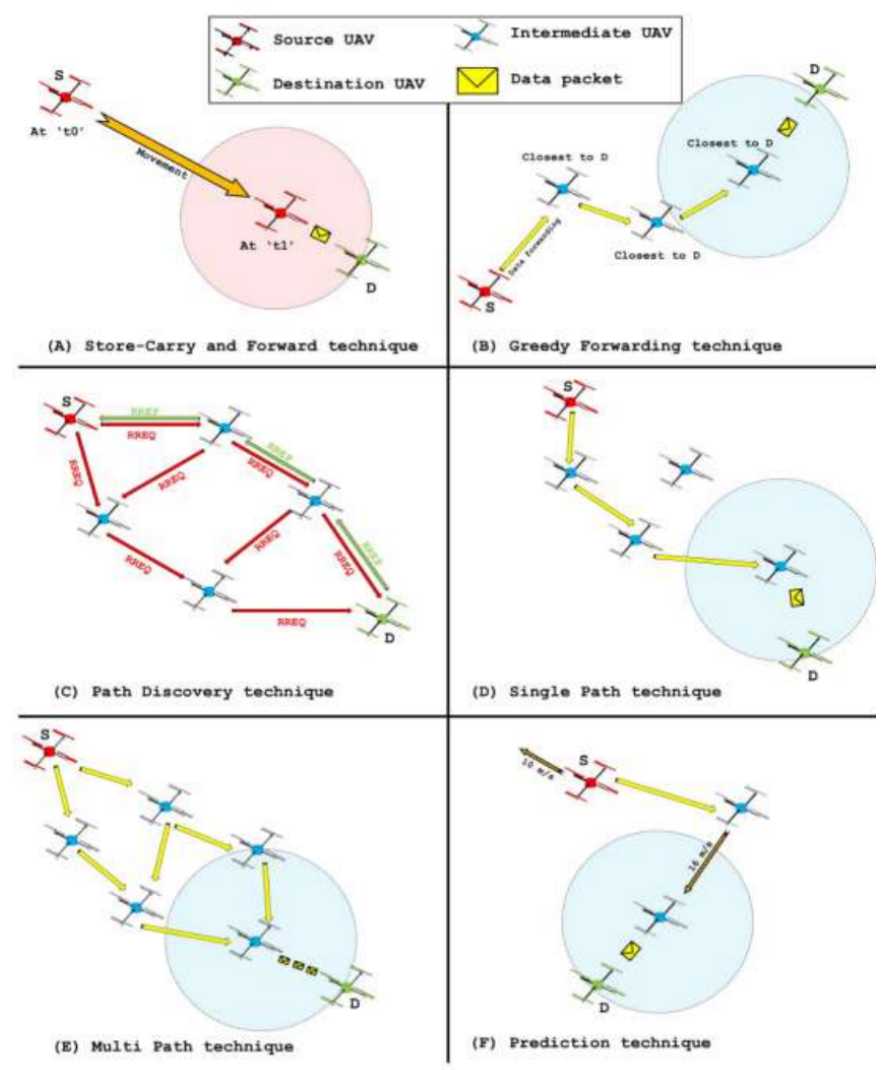

Fig. 3. A Manhattan Grid Topology with Two-Way Roads.

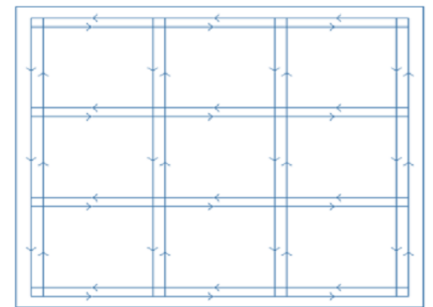

Fig. 4. A Random Waypoint Model.

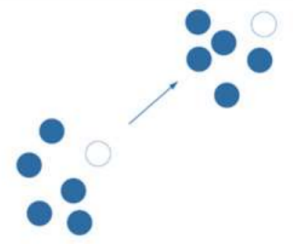

Fig. 5. An Example of a Pursue Mobility Model.
(1)

(2)

(3)

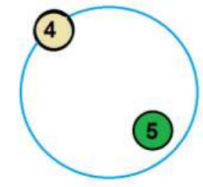

Fig. 6. An Example of a SCRM Model.

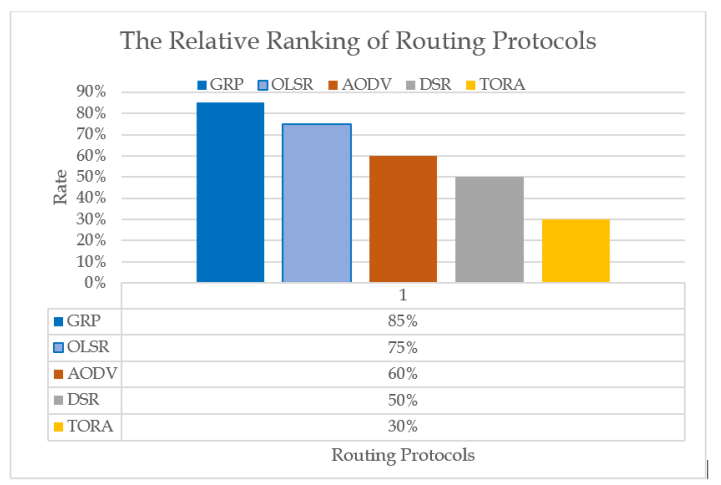

Fig. 7. The Relative Ranking of Routing Protocols in Scenario 1.

\section{Performance Evaluation}

modification, we will use the IEEE 802.11n standard, which allows the use of quality of service in wireless networks.
The present study has focused on the performance evaluation on the basis of mobility, congestion and the reliability of 


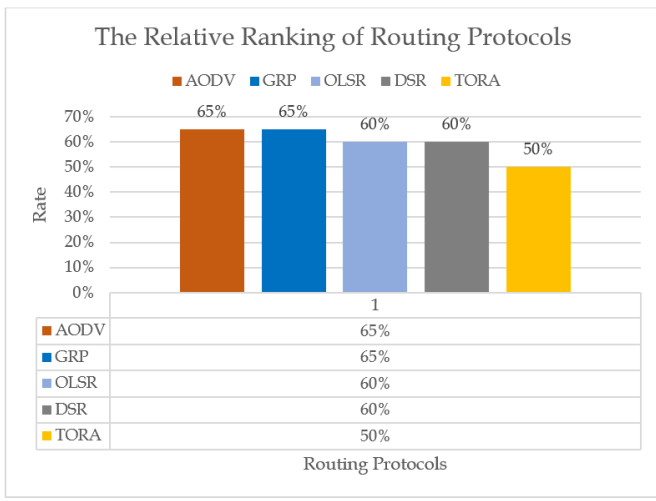

Fig. 8. The Relative Ranking of Routing Protocols in Scenario 2.

the route of the routing algorithm used to establish communications. The mobility model defines the speed, start time and stop time of nodes in network. The trajectory of movement was selected as 2000 meters at maximum. It is essential to perceive the mobility configuration to estimate mobility model of nodes. The movement of UAVs is controlled with parameters such as start and stop time, pause time and speed. The experiments are designed in such a way that performance of routing protocols is assessed using different mobility models in one sender one receiver, one sender all receivers and all senders and all receivers scenarios. A comparison is also performed among all routing protocols.

1) Scenario \#1: One sender to one receiver situation: In our simulation parameter (One to one) in scenario 1 , the numbers of UAVs are 15 and have fixed sender/receiver. The time taken of the simulation is 600 seconds, CBR traffic and data payload of 1024 bytes/packet. All routing protocols are evaluated and compared using performance parameters.

The simulated results show that shown in Fig. 7 indicate comparative ranking of routing protocols in our simulation results. It show that routing protocols can differ related to mobility model. The comparative ranking relies on the UAV speed because of the existence of the mobility that indicates frequent link failures. Each routing protocol plays an important role when link fails. Unlike OLSR, AODV and DSR, the Hybrid protocol GRP shows stable performance for all mobility models. TORA shows high delay and packet loss ratio in most scenarios. The algorithm of TORA keeps the "direction of the next destination" for packet forwarding. As a result, the source node follows downstream paths to the destination between several nodes. This will not be utilized if source node does not require path information before any topological changes.

2) Scenario \# 2: All sender to one receive : In scenario 2 simulation, 15 nodes can be sent traffic to the base station. The speed of UAVs fluctuates between 5 and $40 \mathrm{~m} / \mathrm{sec}$. The simulation time is $600 \mathrm{~s}$, data payload of 1024 bytes/packet and CBR traffic.

Fig. 8 provide detailed results for scenario 2. The Reactive (AODV) and the Hybrid (GRP) have the same record with all existing mobility models. TORA shows higher delay in most scenarios.
3) Scenario \#3: All senders to All receiver situation: In simulation scenario 3 , the numbers of UAVs are 15 and every node can be treated as a source, destination, or routing node. The duration of simulation is 600 seconds, and traffic type considered is CBR. Regarding performance of different mobility moadels as shown in Fig. 9, we find that DSR and TORA have comparatively the lowest throughput. Finally, it is found that OLSR performs best in terms of throughput. It can be noticed that GRP and AODV are similar to each other in values fortunately.

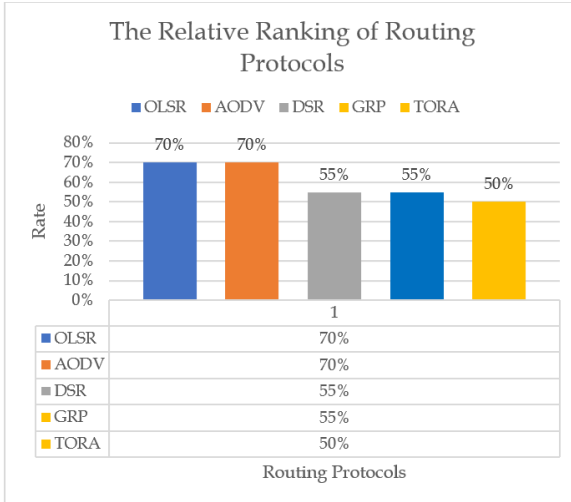

Fig. 9. The Relative Ranking of Routing Protocols in Scenario 3.

Now, we evaluate all mobility models based on performance parameters including delay, throughput, drop ratio and number of hops. Fig. 10 (a) shows performance of all protocol for PRS mobility model. It shows average result of all three scenarios mentioned above. Using PRS mobility model, TORA shows high delay and number of hops. MGM mobility model is evaluated in Fig. 10 (b). DSR routing protocols takes more time for end-to-end transmission and number of hops as compared to other protocols but provides maximum throughput and least packet drop ratio. AODV routing protocol takes minimum time for transmission and number of hops are slightly less than DSR, drop ratio is higher than DSR but less than other protocols which results in lesser throughput than DSR but higher throughput as compared to other protocols.

Using RWPM mobility model, DSR routing protocol outperforms other protocols as shown in Fig. 10 (c). Whereas after DSR, AODV performs really well. DSR has maximum throughput and least packet drop ratio although delay and number of hops a bit higher than other protocols.

Fig. 10 (d) shows performance of all protocol for SCRM mobility model. It shows average result of all three scenarios mentioned above. Using SCRM mobility model, DSR and AODV show high throughput. AODV shows minimum endto-end delay and DSR shows minimum packet drop ratio.

\section{CONCLUSION AND Future WORK}

Depending on the simulation results, the effect of node movement on performance is higher than the effect of node speed on performance. OLSR (proactive protocol) and GRP (hybrid protocol) are stable, TORA (hybrid protocol) is vulnerable and AODV/DSR (reactive protocols) are moderate in nature. It is noticed that node mobility results in rapid topology changes which affects network performance more than speed. 

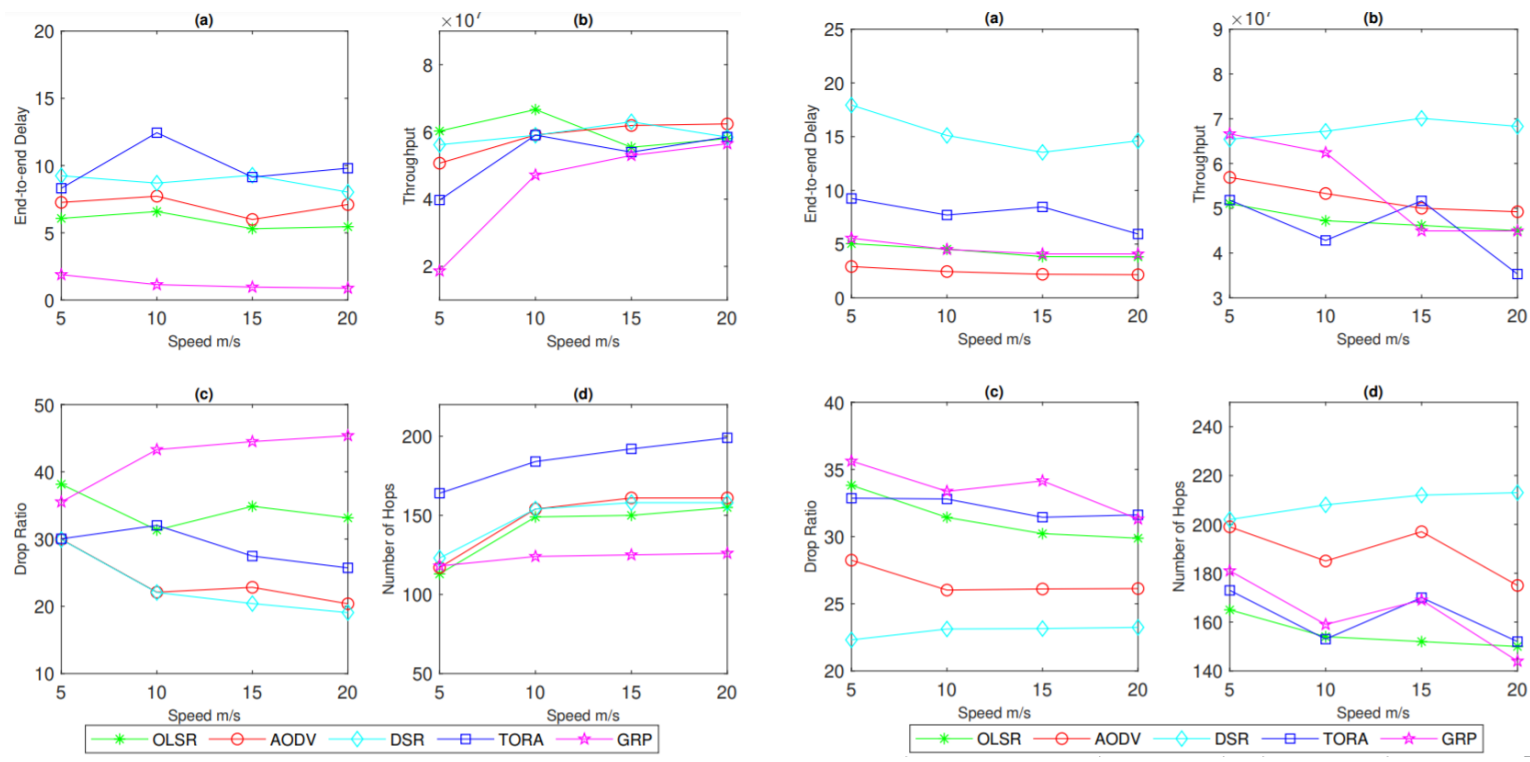

(a) Performance Evaluation of all scenarios with PRS Mobility Model

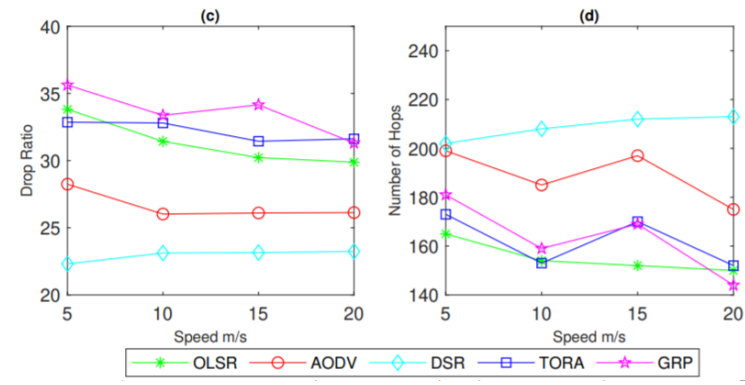

(b) Performance Evaluation of all scenarios with MGM Mobility Model
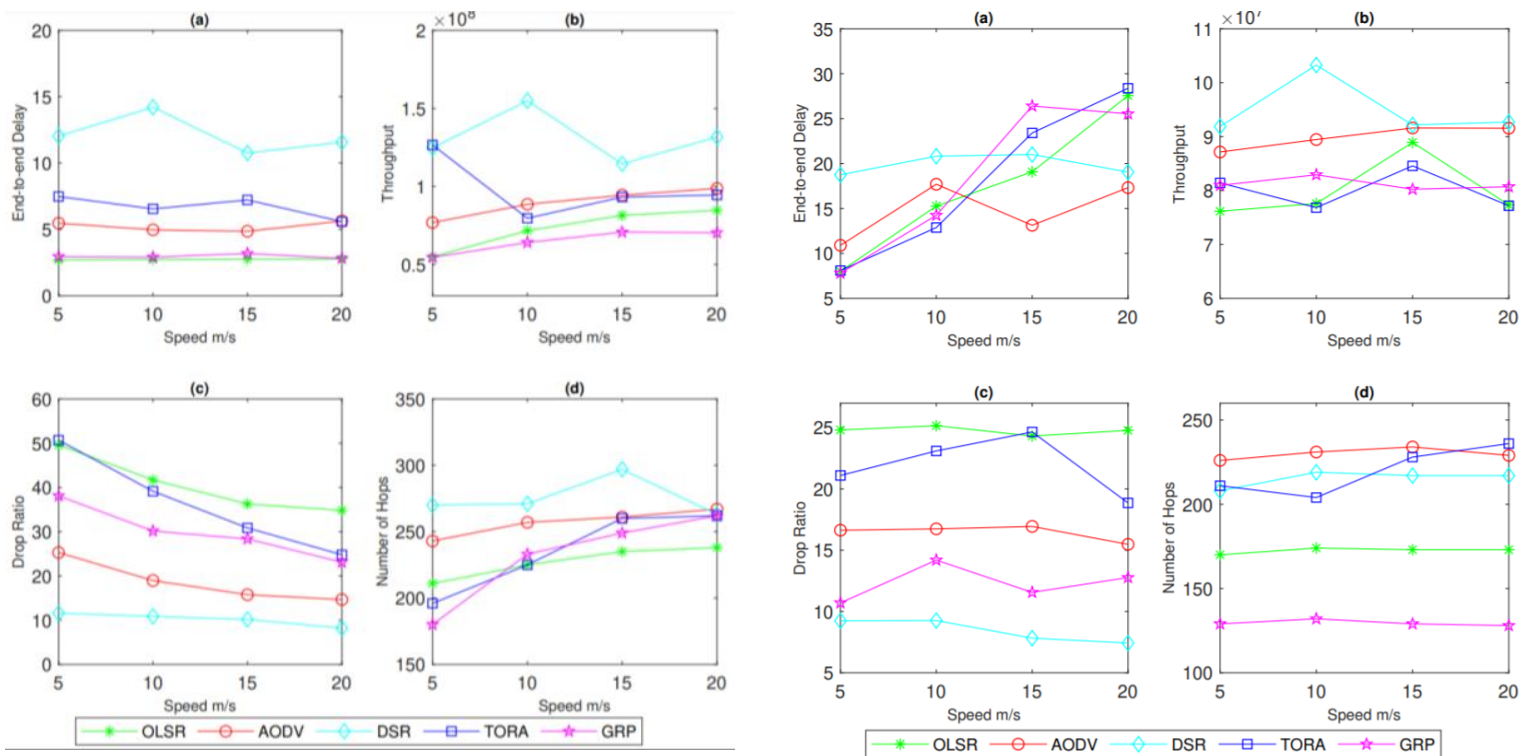

(c) Performance Evaluation of all scenarios with RWPM Mobility Model

(d) Performance Evaluation of all scenarios with SCRM Mobility Model

Fig. 10. Performance Evaluation of Different Mobility Models and Routing Protocols.

Each mobility model undergoes topology changes differently. Among four mobility models, SCRM is the most competitive in high-speed environments. OLSR outperforms using MGM and PRS mobility models than other mobility models. While designing routing protocols, it is wise to make appropriate improvements on existing ones according to specific needs.

In addition, we discussed routing protocols along with their working and limitations. We also have provided a brief qualitative review of the aforementioned routing protocols on the basis of significant parameters. All these factors affect the performance of FANETs. We also have provided a simulation- based study of the topology-based routing protocols.

There are specific restrictions of FANETs because of its characteristics such as dynamic topology, security, limited bandwidth and UAVs management. One other problem is routing in swarm behaviour within FANETs. Because they work with each other to create a network, how they interact together also has importance in communication. The future work involve routing protocol depending on the swarm intelligence which will be helpful in setting efficient routing protocols for FANET environment in $3 D$ - space mobility of UAVs. 


\section{ACKNOWLEDGMENT}

This work is supported by grant number 43208008, Deanship of Scientific Research (DSR) of Umm al Qura University, Kingdom of Saudi Arabia. The authors would like to express their gratitude for the support and generous contribution towards pursuing research in this area

\section{REFERENCES}

[1] G. Pajares, "Overview and current status of remote sensing applications based on unmanned aerial vehicles (uavs)," Photogrammetric Engineering \& Remote Sensing, vol. 81(4), p. 281-329, 2015.

[2] George, Joel, P. B. Sujit, and João B. Sousa, "Search strategies for multiple UAV search and destroy missions," J. Intell. Robot. Syst., vol. 61, p. 355-367, 2011.

[3] Choudhary, Gaurav, et al, "Internet of Drones (IoD): Threats, Vulnerability, and Security Perspectives," in The 3rd International Symposium on Mobile Internet Security, At Cebu, Philippines, 2018.

[4] M. A. Khan, A. Safi, I. M. Qureshi, and I. U. Khan, "Flying ad-hoc networks (FANETs):A review of communication architectures, and routing protocols," in IEEE First International Conference on Latest trends in Electrical Engineering and Computing Technologies (INTELLECT), Karachi, Pakistan, 2017.

[5] Bujari, Armir and et al, "Flying ad-hoc network application scenarios and mobility models," International Journal of Distributed Sensor Networks, vol. 1550147717738192, 2017.

[6] Pandit, Krushna J., and Shah Niyati, "Topology control in MANET for efficient energy conservation," International Journal of Advanced Computer Research, vol. 3.2, p. 108, 2013.

[7] Kaur, Kulwinder, and Barinderpal Singh, "Survey analysis of routing protocols and mobility models in manets," International Journal of Advanced Science and Technology, vol. 85, pp. 55-64, 2015.

[8] Patel, S., Patel, H., Patel, S., and Patel, V, "A Study of Topology control in MANET," Presentado en SIEICON, International Journal of Advance Engineering and Research Development, p. 1-4, 2017.

[9] Robinpreet Kaur and Mritunjay Kumar Rai, "A Novel Review on Routing Protocols in MANETs," Undergraduate Academic Research Journal, vol. 1, no. 1, p. 2278 - 1129, 2012.

[10] Sarkar, Subir Kumar, Tiptur Gangaraju Basavaraju, and C. Puttamadappa, "Ad-hoc mobile wireless networks: principles, protocols, and applications," in CRC Press, 2016.

[11] J. e. a. Liu, "A survey on position-based routing for vehicular ad hoc networks," in Telecommunication Systems, 2016, pp. 15-30.

[12] O. K. Sahingoz, "Networking models in flying ad-hoc networks (FANETs): Concepts and challenges," Journal of Intelligent \& Robotic Systems, Vols. 74(1-2), pp. 513-527, 2014.

[13] Maistrenko, Vasily A., Leonov V. Alexey, and Volkov A. Danil., "Experimental estimate of using the ant colony optimization algorithm to solve the routing problem in," in International Siberian Conference on Control and Communications (SIBCON)., IEEE, 2016.

[14] Leonov, Alexey V., and George A. Litvinov, "Applying aodv and olsr routing protocols to air-to-air scenario in flying ad hoc networks formed by mini-uavs,"in 2018 Systems of Signals Generating and Processing in the Field of on-Board Communications, IEEE, 2018.

[15] Nayyar and Anand, "Flying Adhoc Network (FANETs): Simulation Based Performance Comparison of Routing Protocols: AODV, DSDV, DSR, OLSR, AOMDV and HWMP," in 2018 International Conference on Advances in Big Data, Computing and Data Communication Systems (icABCD), IEEE, 2018.

[16] Yadav, Krishan, Amit Nain, and Sunil Maakar, "Routing protocols in FANET: Survey," in Proceedings of National Conference on Innovative Trends in Computer Science Engineering, (ITCSE-2015), ISSN, 2015.

[17] Nayyar, Anand, and Rajeshwar Singh, "Ant colony optimization-computational swarm intelligence technique," in 2016 3rd International conference on computing for sustainable global development (INDIACom). IEEE, 2016.

[18] Nayyar, Anand, and Rajeshwar Singh, "A comprehensive review of ant colony optimization (ACO) based energy-efficient routing protocols for wireless sensor networks," International Journal of Wireless Networks and Broadband Technologies (IJWNBT), vol. 3.3, pp. 33-55, 2014.

[19] Jailton, J., Carvalho, T., Araújo, J., and Francês, R, "Relay Positioning Strategy for Traffic Data Collection of Multiple Unmanned Aerial Vehicles Using Hybrid Optimization Systems," A FANET-Based Case Study, p. 11, 2017.

[20] Liu, Kesheng, Jun Zhang, and Tao Zhang, "The clustering algorithm of UAV networking in near-space," in 2008 8th International Symposium on Antennas, Propagation and EM Theory, IEEE, 2008.

[21] Maistrenko, Vasily A., Leonov V. Alexey, and Volkov A. Danil, "Experimental estimate of using the ant colony optimization algorithm to solve the routing problem in FANET," in In Control and Communications (SIBCON), 2016 International Siberian Conference on, 2016.

[22] Bendale, Lubdha M., Roshani L. Jain, and Gayatri D. Patil, "Study of various routing protocols in mobile ad-hoc networks," International Journal of Scientific Research in Network Security and Communication, vol. 6.01 , pp. 1-5, 2018.

[23] D. e. a. Zhang, "Novel dynamic source routing protocol (DSR) based on genetic algorithm-bacterial foraging optimization," International Journal of Communication Systems, vol. e3824, 2018.

[24] Prasanth K. and Sivakumar P, "Location Based Routing Protocol - A Survey," in International Conference on Computer Communication and Informatics (ICCCI 2014), 2014 .

[25] Bruzgiene, Rasa, Lina Narbutaite, and Tomas Adomkus, "MANET network in internet of things system," in Ad Hoc Networks, 2017, p. 89.

[26] Jubair, Mohammed Ahmed, et al, "Performance Evaluation of Ad-Hoc On-Demand Distance Vector and Optimized Link State Routing Protocols in Mobile Ad-Hoc Networks," International Journal on Advanced Science, Engineering and Information Technology, pp. 1277-1283, 2018.

[27] F. Bai and A. Helmy, "A Survey of Mobility Models in Wireless Adhoc Network," in University of Southern California , USA, 2004.

[28] Vijayavani, G. R., and G. Prema. , "Performance comparison of MANET routing protocols with mobility model derived based on realistic mobility pattern of mobile nodes," in 2012 IEEE International Conference on Advanced Communication Control and Computing Technologies (ICACCCT), IEEE, 2012.

[29] K Kumari, S Maakar, B sah, "A Survey: Different Mobility Model for FANET," International Journal of Advanced Research in Computer Science and Software Engineering, 2015.

[30] R. Technology, "OPNET," 2019 . [Online]. Available: https://www.riverbed.com/gb/products/steelcentral/opnet.html. 